\title{
Robot-assisted laparoscopic transperitoneal pelvic lymphadenectomy and metastasectomy for melanoma: initial report of two cases
}

\author{
William Sohn • David S. Finley • James Jakowatz • \\ David K. Ornstein
}

Received: 13 March 2010/Accepted: 26 April 2010/Published online: 11 June 2010

(C) The Author(s) 2010. This article is published with open access at Springerlink.com

\begin{abstract}
Robotic pelvic lymphadenectomy is a well established procedure in the urologic and gynecologic literature. To our knowledge robotic pelvic lymphadectomy for metastatic melanoma has yet to be described. Herein we present the first report of robot-assisted pelvic lymphadenectomy in malignant melanoma. After placement of six laparoscopic ports $(12 \mathrm{~mm}$ camera, three $8-\mathrm{mm}$ robotic ports, $12-\mathrm{mm}$ and $5-\mathrm{mm}$ assistant ports) the DaVinci $\mathrm{S}$ robot (Intuitive Surgical, CA, USA) was docked in standard fashion with the patient in low lithotomy. In both cases the patients had enlarged pelvic lymph nodes on computed tomography and complete excision of these masses was accomplished along with complete lymphadenectomy extending from Cooper's
\end{abstract}

\section{W. Sohn $(\square)$}

Department of Urology, University of California, Irvine, Orange, CA 92868, USA

e-mail: wsohn@hotmail.com

\section{S. Finley}

Department of Urology, David Geffen School of Medicine, University of California, Los Angeles, Los Angeles, CA 90024, USA

e-mail: dfinley@mednet.ucla.edu

\section{J. Jakowatz}

Department of Surgery, University of California,

Irvine, Orange, CA 92868, USA

e-mail: jgjakowa@uci.edu

\section{K. Ornstein}

Naples Community Hospital, 733 4th Avenue,

North Naples, FL 34102, USA

e-mail: dornstein@rtsx.com ligament to just below the hypogastric artery in case 1 and to level of the bifurcation of aorta in case 2. A PK Maryland Dissector and monopolar scissors were used for dissection. Both patients were discharged on postoperative day \#1. Robotic pelvic lymphadenectomy can be safely used for management of patients with metastatic melanoma involving the pelvic lymph nodes. Compared with the standard open procedure, pelvic lymphadenectomy with robotic assistance is associated with excellent vision and minimum morbidity.

Keywords Robotic - Lymphadenectomy - Melanoma . Laparoscopic · Minimally-invasive surgery $\cdot$ Metastasis · Metastatectomy

\section{Introduction}

Cutaneous melanoma is traditionally managed with wide local excision down to the deep fascia [1]. For higher-risk lesions based on pathologic features including Clark and Breslow criteria, lymphatic mapping with sentinel lymph node biopsy is performed to detect regional nodal disease and determine the need for more aggressive lymphadenectomy. In the groin this may include superficial and deep inguinal lymph node dissection [2]. Pelvic node dissection for melanoma and other malignancies (i.e. prostate, bladder, penis) has been accomplished via an open and laparoscopic route [3]. In addition, in some cases, limited sites of distant metastatic disease occur which may be amenable to surgical excision for curative purposes or prevention of morbidity from local tumor invasion [4-6]. Herein we present the first report, to our knowledge, of two cases of RPL and excision of isolated pelvic melanoma metastases. 


\section{Case presentation}

Case 1

The patient is a 50-year-old man who first presented in 2006 with a changing pigmented lesion on his right upper medial calf below the knee. On biopsy, this lesion was found to be a 16-mm Clark level V melanoma with focal ulceration and a high mitotic rate. The patient presented with palpable right inguinal lymph nodes. A fine needle aspirate showed only reactive lymphocytes. Metastatic work-up included full body PET/CT scan and MRI of the brain which showed no evidence of metastatic disease. A wide local excision of the lesion with $2-\mathrm{cm}$ margins and sentinel lymph node biopsy was performed. Pathology showed malignant melanoma measuring 2-mm in greatest diameter with negative surgical margins and lymph nodes. Subsequently, the patient presented eleven months later with a mass on the superior aspect of the surgical excision site, and a palpable inguinal lymph node. Fine needle aspirate of the palpable node was positive for malignant melanoma. The patient underwent a wide local excision of the recurrent lesion, and open right inguinal lymph node dissection. Pathology again showed malignant melanoma, with clear proximal and distal margins, and 20 of 20 nodes positive for metastatic melanoma. He was started on systemic therapy with G-CSF for five months. PET/CT scan showed a $4.2 \mathrm{~cm}$, right iliac nodal mass with central necrosis. The decision was made to pursue RPL and metastasectomy.

\section{Case 2}

A 53-year-old women initially underwent a wide local excision of a $5.5 \times 4.0 \mathrm{~cm}$ melanoma over the left fibular head in 2002 with hemigastrocnemius muscle flap and split thickness skin graft from the buttock for coverage. Concurrent with this procedure, Lymphazurin injection and gamma probe excision of three superficial inguinal sentinel lymph nodes showed counts of 1,700 per second. Pathology revealed nodular type malignant melanoma (Clark V, Breslow $19 \mathrm{~mm}$ ) with negative margins; 1 of 3 lymph nodes were positive for micrometastatic disease. Given a negative metastatic work-up and consultation with hematology-oncology the patient elected at that time for no further treatment. In 2007, she recurred approximately $10 \mathrm{~cm}$ distant from the initial lesion. Metastatic work-up with a PET scan was negative. Lymphoscintigraphy was performed with $1 \mathrm{mCi}$ of filtered technetium $99 \mathrm{~m}$ sulfur colloid which identified migration of radioactivity to two left inguinal lymph nodes. She underwent repeat wide local excision and sentinel lymph node mapping with counts in excess of 15,000 per second in the region of the inguinal ligament. The primary lesion again was malignant melanoma (Clark V and Breslow $3 \mathrm{~mm}$ ) with negative margins and 4 of 8 nodes involved with disease. She again declined any further adjuvant therapy. In 2008, she was found to have a $5.0 \times 3.0 \mathrm{~cm}$ left external iliac lymph node on CT scan and underwent CT-guided biopsy of left obturator chain lymph node revealing metastatic melanoma. A robotassisted laparoscopic left pelvic lymph node dissection with metastasectomy and deep inguinal groin dissection were performed.

\section{Methods}

Between January 2007 and July 2008 two patients underwent robotic excision of metastatic melanoma with pelvic lymphadenectomy. After Institutional Review Board approval (exemption), the medical records were reviewed for clinical, operative, and pathologic data.

\section{Technique}

The patients were placed on spreader bars in exaggerated Trendelenburg position. A Foley catheter was placed. Pneumoperitoneum to $20 \mathrm{~mm} \mathrm{Hg}$ was established with closed Veress needle insertion just above the umbilicus. A $12-\mathrm{mm}$ port was placed above the umbilicus, two $8-\mathrm{mm}$ robotic ports were placed lust lateral to the medial umbilical ligaments approximately $16 \mathrm{~cm}$ from the pubic symphysis and $10 \mathrm{~cm}$ from the midline. A third 8-mm robotic port was placed for the fourth robotic arm on the left side $10 \mathrm{~cm}$ lateral to the left sided $8-\mathrm{mm}$ robotic port. A $12-\mathrm{mm}$ assistant port was placed in the right lower quadrant and a 5 -mm port in the right upper quadrant two finger breadths below the costal margin $2 \mathrm{~cm}$ medial to the $8 \mathrm{~mm}$ port (Fig. 1). The DaVinci $\mathrm{S}$ robot was docked and pneumoperitoneum was lowered to $12 \mathrm{~mm} \mathrm{Hg}$.

\section{Case 1}

The ureter was identified as it crossed-over the external iliac artery and the posterior peritoneum was incised lateral to this and medial to the artery. The ureter was mobilized and the mass was identified inferomedially. The mass was adherent to the external iliac vein and extended down on to the obturator nerve (Fig. 2). The medial border of the external iliac vein was exposed. The external iliac vein was retracted laterally with a $10 \mathrm{~mm}$ Kitner and the mass was circumscribed and separated from the vein using monopolar cautery, argon beam coagulation, and $5 \mathrm{~mm}$ clips. The vas deferens was identified and transected using bipolar cautery to improve access to the iliac-obturator nodes. The obturator nerve was identified and separated 


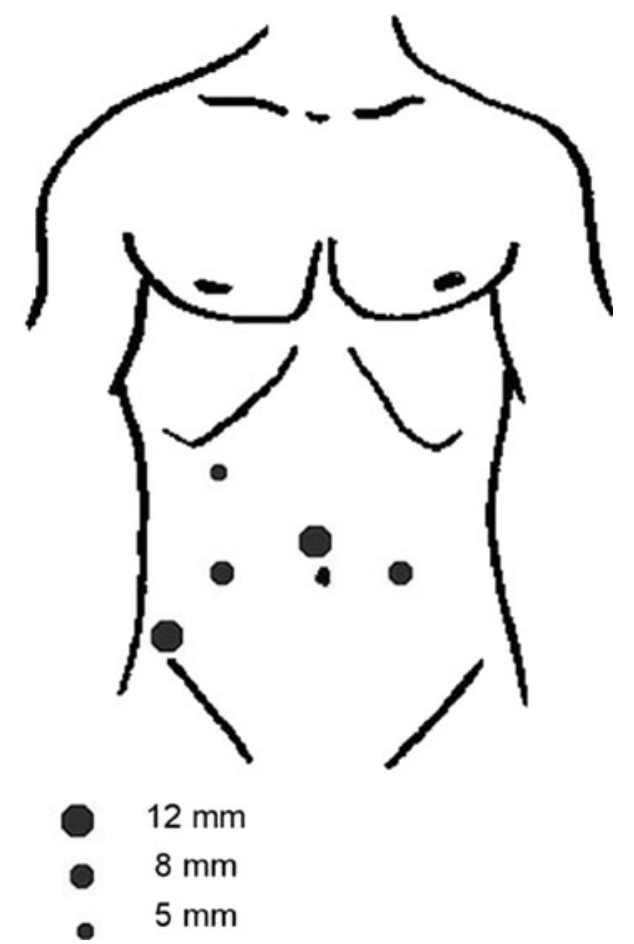

Fig. 1 Schematic diagram of port placement

from the mass. The tissue from the obturator fossa up to the common iliac artery was removed. The obturator lymph node tissue and mass were removed en bloc and placed in a $10 \mathrm{~mm}$ Endocatch bag (Covidien, CA, USA). After copious irrigation with sterile water and observation for hemostasis under $2 \mathrm{~mm} \mathrm{Hg}$ pneumoperitoneum, Floseal (Baxter, CA, USA) and Surgicel (Ethicon, NJ, USA) were placed along the resection bed and the specimen was removed through the supraumbilical port site after extending the incision to $3 \mathrm{~cm}$.

Satisfactory pain control was achieved with $30 \mathrm{mg}$ IV Toradol every $6 \mathrm{~h}$ and Tylenol. The patient was discharged home on postoperative day \#1 in good condition with minimal pain. At one week follow-up the incisions were healing well and the patient was tolerating light physical activity with no narcotics.
Case 2

Adhesions were taken down from the colon and the line of Toldt was incised sharply and then the colon was reflected medially. The external iliac artery was identified and the lymph node packet between the artery and the vein was dissected up to the bifurcation of the common iliac artery. The mass in the obturator fossa (Fig. 3) was identified and dissected out using monopolar and bipolar cautery and $5 \mathrm{~mm}$ clips. The obturator nerve was identified and preserved. The packets were placed in $10 \mathrm{~mm}$ Endocatch bags. After observation for bleeding under $2 \mathrm{~mm} \mathrm{Hg}$ pneumoperitoneum, Floseal was placed in the dissection bed and the specimens were removed through the midline port site after extending the incision slightly. Immediately following this procedure she underwent a deep inguinal lymphadenectomy including removal of the saphenous vein. Final pathology from the groin dissection revealed $3 / 3$ positive nodes, $4 / 15$ positive nodes from the external iliac artery, and obturator lymph node chains.

On postoperative day 1 the patient was discharged home tolerating a regular diet with pain well controlled by Toradol and Vicodin. On one week follow-up the surgical incisions were healing well and she was tolerating light physical activity. She currently is undergoing evaluation for adjuvant therapy.

\section{Discussion}

Although the role of pelvic lymphadenectomy is well established in the management of urologic and gynecologic malignancies, its role in staging and management of malignant melanoma continues to evolve. Contemporary indications for deep pelvic lymphadenectomy include:

1 four or more positive superficial lymph nodes;

2 radiological imaging or clinico-pathological evidence of deep pelvic node involvement; or

3 confirmed metastatic disease within the node of Cloquet [7].
Fig. 2 Contrast enhanced CT of the pelvis showing right obturator mass (arrow) in axial (a) and coronal planes (b)
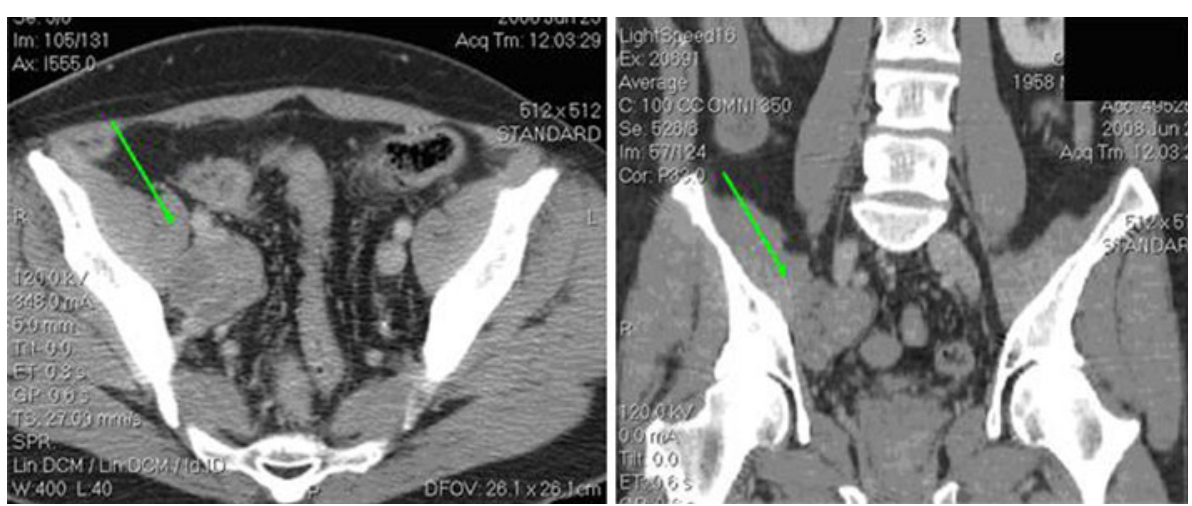


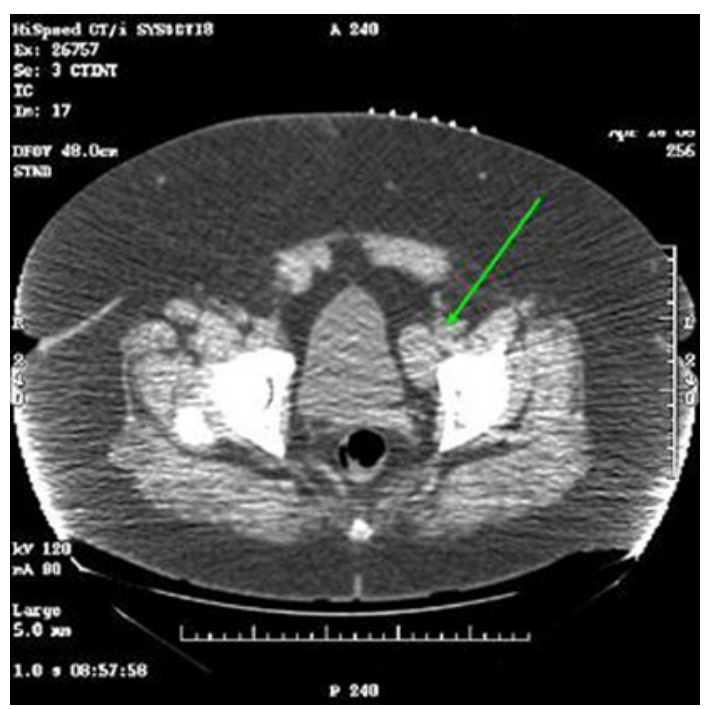

Fig. 3 Contrast enhanced CT of the pelvis showing left obturator mass (arrow) in axial plane

In a recent review by Badgwell and colleagues [7] of patients who underwent deep pelvic lymph node dissection, the presence of three or fewer positive deep pelvic lymph nodes was associated with increased cancer-specific survival over five years.

For any procedure, particularly for staging purposes, the risk benefit analysis is critical. Much of the morbidity of traditional open pelvic lymphadenectomy is due to incisional complications. Standard laparoscopy was introduced in an effort to reduce this morbidity [8]. The advantages of a minimally invasive approach in pelvic lymph node dissection have been well documented in the literature and include accelerated healing time, shortened postoperative disability period, less incisional pain, and reduced scarring [9].

Although the benefits of laparoscopic lymphadenectomy have been established, the technical challenges, particularly in cases with enlarged metastatic lymph nodes, have limited its widespread application. The introduction of a robotic interface has shortened the learning curve and dramatically expanded the acceptance of complex, less invasive (laparoscopic) surgical procedures [10]. The benefit of robot assistance for radical prostatectomy with pelvic lymph node dissection has been widely described in the literature [11]. The magnification that the robot enables and the 3D visual capacity aid in meticulous dissection of the tissue, which may increase nodal yield and reduce operative morbidity. Robot assistance incorporates the minimally invasive benefits of classic laparoscopy with the additional benefits of increased visualization and dexterity.

\section{Conclusions}

RPL for metastatic melanoma can be performed safely. The exact role of RPL in malignant melanoma remains to be determined. This initial report supports further study into the application of robotics to the management of metastatic melanoma in the pelvis.

Acknowledgments We appreciate Dr Hak Lee, MD, for reviewing this manuscript.

Open Access This article is distributed under the terms of the Creative Commons Attribution Noncommercial License which permits any noncommercial use, distribution, and reproduction in any medium, provided the original author(s) and source are credited.

\section{References}

1. Holmström H (1992) Surgical management of primary melanoma. Semin Surg Oncol 8(6):366-369

2. Patel JK, Didolkar MS, Pickren JW, Moore RH (1978) Metastatic pattern of malignant melanoma. A study of 216 autopsy cases. Am J Surg 135:807

3. Picciotto F, Volpi E, Zaccagna A et al (2003) Transperitoneal laparoscopical iliac lymphadenectomy for treatment of malignant melanoma. Surg Endosc 17(10):1536-1540

4. Karakousis CP, Velez A, Driscoll DL, Takita H (1994) Metastasectomy in malignant melanoma. Surgery 115(3):295-302

5. Ollila DW, Hsueh EC, Stern SL, Morton DL (1999) Metastasectomy for recurrent stage IV melanoma. J Surg Oncol 71(4):209-213

6. Overett TK, Shiu MH (1985) Surgical treatment of distant metastatic melanoma. Indications and results. Cancer 56(5):12221230

7. Badgwell B, Xing Y (2007) Pelvic lymph node dissection is beneficial in subsets of patients with node-positive melanoma. Ann Surg Oncol 14(10):2867-2875 Epub 2007 Aug 1

8. Mommsen S (1995) Open versus laparoscopic diagnostic pelvic lymphadenectomy. Scand J Urol Nephrol Suppl 172:23-25

9. Eichel L, McDougall EM, Clayman RV (2007) Basics of laparoscopic urologic surgery, 9th edn. Campbell-Walsh Urology, Saunders, Wein

10. Rozet F, Harmon J, Cathelineau X et al (2006) Robot-assisted versus pure laparoscopic radical prostatectomy. World J Urol 24(2):171-179

11. Ahlering TE, Skarecky D, Lee D, Clayman RV (2003) Successful transfer of open surgical skills to a laparoscopic environment using a robotic interface: initial experience with laparoscopic radical prostatectomy. J Urol 170(5):1738-1741 